\title{
Lifshitz metric with hyperscaling violation from NS5-D $p$ states in string theory
}

\author{
Parijat Dey 1 and Shibaji Roy 2 \\ Saha Institute of Nuclear Physics, 1/AF Bidhannagar, Calcutta-700 064, India
}

\begin{abstract}
In previous papers [1, 2] we have shown how Lifshitz-like space-time (space-time having a Lifshitz scaling along with a hyperscaling violation) can arise by taking near horizon limits of certain intersecting solutions (F-string with $\mathrm{D} p$-branes and also with two D-branes) of string theory. In this paper we construct intersecting bound state solutions in the form of NS5-D $p$-branes (with $1 \leq p \leq 6$ ) of type II string theories. These are 1/4 BPS and threshold bound states unlike the known NS5-D $p$ bound states which are 1/2 BPS and non-threshold. We show that the near horizon limits of these solutions also lead to Lifshitz-like space-time with the dynamical scaling exponent $z=0$ and the hyperscaling violation exponent $\theta=9-p$. The spatial dimension of the boundary theory is $d=7-p$. The dilatons in these theories are not constant in general (except for $p=5$ ) and therefore produce RG flows. So, we also consider the strong coupling phases of these theories and find that these phases also have similar Lifshitz-like structures, except for $p=2$, where it has an $\mathrm{AdS}_{3}$ structure.
\end{abstract}

\footnotetext{
${ }^{1}$ E-mail: parijat.dey@saha.ac.in

${ }^{2}$ E-mail: shibaji.roy@saha.ac.in
} 


\section{Introduction}

It is well-known that string theory admits various kinds of spatially extended solutions, generically called branes, which preserve certain fraction of space-time supersymmetries. These solutions may be constituted of a single type of brane or a composite of more than one type of branes. They have been proved quite useful as string theory in the near horizon geometry (a kind of low energy limit) of a stack of coincident branes can be seen to be holographically dual to a theory without gravity living on the boundary. This is a strongweak duality and so one can gain insight about the strongly coupled field theory by studying the weakly coupled string or supergravity theory [3, 4, 5, 6]. For D3-brane this is the AdS/CFT correspondence of Maladacena [3] where the boundary theory is four dimensional, $\mathcal{N}=4, \mathrm{SU}(N)$ super Yang-Mills theory (which is conformal). Similar correspondence is also believed to hold for other types of branes which goes by the name gauge/gravity duality [6]. So, for example, for $\mathrm{D} p$-branes, the boundary theory is $(p+1)$-dimensional super Yang-Mills theory (which is non-conformal) with 16 supercharges [7].

Similar correspondence holds good even for the composite brane states. Composite brane solutions also known as intersecting branes could be of different types. So, for example, there are 1/2 BPS non-threshold types, such as, $\mathrm{D}(p-2)-\mathrm{D} p$ [8, 9, 10] (for $1 \leq p \leq 6$ ), F-D $p$ [11] (for $1 \leq p \leq 7$ ) and NS5-D $p$ [12, 13, 14] (for $0 \leq p \leq 5$ ) among others. In the near horizon limit (or decoupling limit) they give rise to some non-gravitational, non-local theories on the boundary. For $\mathrm{D}(p-2)-\mathrm{D} p$, we get noncommutative Yang-Mills (NCYM) theory in $(p+1)$-dimensions [15, 16], for $\mathrm{F}-\mathrm{D} p$ we get $(p+1)$-dimensional noncommutative open string (NCOS) theory [17, 18] and for NS5-D $p$ we get six dimensional open D $p$-brane (OD $p$ ) theory [19. So, one can gain insight about these non-local theories, in the strongly coupled regime, by studying the gravity duals. The other type of composite brane solutions are the 1/4 BPS and threshold intersecting brane solutions [20, 21, 22]. These are $\mathrm{D}(p-4)$ - $\mathrm{D} p$ (for $3 \leq p \leq 6)$ and we have also found some new solutions F-D $p$ (for $0 \leq p \leq 5)$ [1], $\mathrm{D}(p-2)-\mathrm{D} p$ (for $3 \leq p \leq 5$ ) and $\mathrm{D} p$ - $\mathrm{D} p^{\prime}$ (for $2 \leq p \leq 4$ ) [2]. All these solutions (except some), as we have seen, give rise to Lifshitz-like space-time in the near horizon limit3. It is known that Lifshitz scaling symmetry, which is a non-relativistic symmetry, arise as a possible symmetry in some condensed matter systems at quantum critical point [40, 41]. So, it may be the case that the space-time we obtain from these intersecting bound state solutions in the near horizon limit are the gravity duals of such condensed matter systems near quantum critical point and since the latter systems are strongly coupled, we can learn about the phase structures by studying the gravity solutions in the spirit of AdS/CFT correspondence.

\footnotetext{
${ }^{3}$ The concept of hyperscaling violation was introduced in the context of random field Ising system in [23]. In gauge/gravity duality this was identified while describing certain metallic states with hidden fermi surface in [24, 25]. More general gravity solutions having Lifshitz scaling alongwith hyperscaling violation have been found and studied in [26, 27, 28, 29, 30, 31, 32, 1, 2, 33, 34, 35, 36, 37, 38, 39.
} 
In this paper we construct another kind of $1 / 4$ BPS threshold intersecting brane solutions of type II string theories in the form of NS5-D $p$ (for $1 \leq p \leq 6$ ) solutions. These solutions in the near horizon limit give rise to Lifshitz-like space-tim 4 and may describe the gravity dual of some condensed matter systems near quantum critical point5 (unlike the standard NS5-D $p$ which in the near horizon limit gives rise to OD $p$ theories [19, 13, 14, mentioned earlier). The construction proceeds from the F-D5 solution we have obtained in [1]. Then taking an S-duality on that solution gives NS5-D1 solution. Further T-dualities along the spatial directions of NS5-brane yield all the NS5-D $p$ solutions which are 1/4 BPS and threshold solutions. Then taking the near horizon limit and going to a suitable coordinate we get the Lifshitz space-time with hyperscaling violation where the dynamical critical exponent we find is $z=0$ and the hyperscaling violation exponent is $\theta=9-p$. The spatial dimension of the boundary theory is given as $d=7-p$. These values of $(z, \theta, d)$ are shown to satisfy the null energy condition (NEC) [29] so that they may give sensible dual theories. We have seen that the dilatons for these solutions are in general non-constant except for $p=5$ and therefore produce RG flows. We also construct the strongly coupled phases of these solutions either by going to the S-dual frame (for type IIB solutions) or by uplifting the solutions to M-theory (for type IIA solutions). These strongly coupled phases also have similar Lifshitzlike structures except for $p=2$, where the M-theory lift of the NS5-D2 solution has $\mathrm{AdS}_{3}$ structure in the near horizon limit.

This paper is organized as follows. In the next section, we give the construction of NS5$\mathrm{D} p$ solutions of type II string theories and show that their near horizon limit gives rise to Lifshitz-like space-time. In section 3 we discuss the RG flows and give the strongly coupled phases of the various solutions obtained in the previous section. Then we conclude in section 4 .

\footnotetext{
${ }^{4}$ Here in order not to confuse the readers we would like to clarify that Lifshitz-like space-times actually arise after the compactifications of the near horizon NS5-D $p$ metrics on $\mathrm{S}^{2} \times \mathrm{T}^{p-1}$ as described in the next section.

${ }^{5}$ Note that the number of transverse directions of the NS5-D $p$-brane solutions we construct here is three for all $p$. So, in analogy with D6-brane (where also the number of transverse directions is three), one might think that the gravity does not decouple [7] in the near horizon limit of our solutions. Therefore, the near horizon limit of NS5-D $p$-brane solutions may not describe the gravity dual of some condensed matter systems. However, this is not true and gravity does decouple for our solutions in the near horizon limit. The simple reason is that we have shown (later) that Lifshitz metric with hyperscaling violation arise from the near horizon limit of our NS5-D $p$ brane solutions after compactification on $\mathrm{S}^{2} \times \mathrm{T}^{p-1}$. This geometry has been recognised [24, 25, 29] to be the gravity dual of some condensed matter systems near the quantum critical point. So, as soon as we get this geometry (note that it is not obvious from which string theory solution we can get this geometry, but obviously not from the solution where gravity is known not to decouple), it automatically implies that gravity gets decoupled at least in lower dimensions. But since decoupling can not occur just by dimesional reduction or compactification, the gravity does decouple even in the ten dimensional near horizon NS5-D $p$ solutions.
} 


\section{$2 \quad$ NS5-D $p$ and Lifshitz-like space-time}

In this section we first construct the 1/4 BPS threshold NS5-D $p$ bound state solution of type II string theories and then by taking the near horizon limit we will show how they lead to Lifshitz-like space-time in a suitable coordinate. For that we will start from the F-D5 solution obtained in eq.(2.6) of [1]. For $p=5$, the F-D $p$ solution given there takes the form,

$$
\begin{aligned}
d s^{2} & =H_{2}^{\frac{1}{2}}\left[-\frac{d t^{2}}{H_{1} H_{2}}+\frac{\sum_{i=1}^{5}\left(d x^{i}\right)^{2}}{H_{2}}+\frac{\left(d x^{6}\right)^{2}}{H_{1}}+d r^{2}+r^{2} d \Omega_{2}^{2}\right] \\
e^{2 \phi} & =\frac{1}{H_{1} H_{2}} \\
B_{[2]} & =\left(1-H_{1}^{-1}\right) d t \wedge d x^{6}, \quad A_{[6]}=\left(1-H_{2}^{-1}\right) d t \wedge d x^{1} \wedge \cdots \wedge d x^{5}
\end{aligned}
$$

Here the two harmonic functions are given as, $H_{1,2}=1+Q_{1,2} / r$, with $Q_{1,2}$ denoting the charges of the F-string and D5-brane respectively. It is clear from (1) that F-string is lying along $x^{6}$ and D5-brane is lying along $x^{1}, \ldots, x^{5}$. Also $B_{[2]}$ is the NSNS form field which couples to F-string and $A_{[6]}$ is the RR 6-form gauge field which couples to D5-brane. Taking an S-duality on this solution we get NS5-D1 solution and has the form,

$$
\begin{aligned}
d s^{2} & =H_{1}^{\frac{1}{2}} H_{2}\left[-\frac{d t^{2}}{H_{1} H_{2}}+\frac{\sum_{i=1}^{5}\left(d x^{i}\right)^{2}}{H_{2}}+\frac{\left(d x^{6}\right)^{2}}{H_{1}}+d r^{2}+r^{2} d \Omega_{2}^{2}\right] \\
e^{2 \phi} & =H_{1} H_{2} \\
A_{[2]} & =\left(1-H_{1}^{-1}\right) d t \wedge d x^{6}, \quad H_{[3]}=-Q_{2} \operatorname{Vol}\left(\Omega_{2}\right) \wedge d x^{6}
\end{aligned}
$$

Like in (11) the metric here is also given in the string frame. $A_{[2]}$ is the RR 2-form which couples to the D-string lying along $x^{6}$ and $H_{[3]}$ is the NSNS magnetic 3-form field strength which couples to NS5-brane lying along $x^{1}, \ldots, x^{5}$. Harmonic functions $H_{1,2}$ remain the same as given above. Now taking T-dualities along the NS5 brane directions we generate all the other NS5-D $p$ solutions which can be written in the following compact form,

$$
\begin{aligned}
d s^{2} & =H_{1}^{\frac{1}{2}} H_{2}\left[-\frac{d t^{2}}{H_{1} H_{2}}+\frac{\sum_{i=2}^{p}\left(d x^{i}\right)^{2}}{H_{1} H_{2}}+\frac{\left(d x^{1}\right)^{2}}{H_{1}}+\frac{\sum_{j=p+1}^{6}\left(d x^{j}\right)^{2}}{H_{2}}+d r^{2}+r^{2} d \Omega_{2}^{2}\right] \\
e^{2 \phi} & =H_{1}^{\frac{3-p}{2}} H_{2} \\
A_{[p+1]} & =\left(1-H_{1}^{-1}\right) d t \wedge d x^{1} \wedge \ldots \wedge d x^{p}, \quad H_{[3]}=-Q_{2} \operatorname{Vol}\left(\Omega_{2}\right) \wedge d x^{1}
\end{aligned}
$$

Here $p=1, \ldots, 6$ and since these solutions are obtained from 1/4 BPS threshold F-D5 solution by the application of S- and T-dualities, these NS5-D $p$ solutions are also 1/4 BPS and threshold. Note that in writing (3) from (2) we have exchanged the coordinates $x^{6} \leftrightarrow x^{1}$ for convenience. Now the $\mathrm{D} p$-brane is along $x^{1}, \ldots, x^{p}$ and NS5-brane is along $x^{2}, \ldots, x^{6}$ and their charges are $Q_{1}$ and $Q_{2}$ respectively. They overlap on a $(p-1)$ brane along $x^{2}, \ldots, x^{p}$. 
The harmonic functions $H_{1,2}$ remain the same as given before. Next, we take a near horizon limit by approximating $H_{1,2} \approx Q_{1,2} / r$ and substitute in the solution (3). By introducing a new coordinate $u^{2}=r$, we can write the NS5-D $p$ solution in the near horizon limit 6 as follows,

$$
\begin{aligned}
d s^{2} & =Q_{1}^{\frac{1}{2}} Q_{2} u\left[-\frac{d t^{2}}{Q_{1} Q_{2}}+\frac{\sum_{i=2}^{p}\left(d x^{i}\right)^{2}}{Q_{1} Q_{2}}+\frac{\left(d x^{1}\right)^{2}}{Q_{1} u^{2}}+\frac{\sum_{j=p+1}^{6}\left(d x^{j}\right)^{2}}{Q_{2} u^{2}}+4 \frac{d u^{2}}{u^{2}}+d \Omega_{2}^{2}\right] \\
e^{2 \phi} & =\frac{Q_{1}^{\frac{3-p}{2}} Q_{2}}{u^{5-p}} \\
A_{[p+1]} & =-\frac{u^{2}}{Q_{1}} d t \wedge d x^{1} \wedge \ldots \wedge d x^{p}, \quad H_{[3]}=-Q_{2} \operatorname{Vol}\left(\Omega_{2}\right) \wedge d x^{1}
\end{aligned}
$$

It is clear from (44) that under the scaling $t \rightarrow \lambda^{0} t, x^{1} \rightarrow \lambda x^{1}, x^{p+1, \ldots, 6} \rightarrow \lambda x^{p+1, \ldots, 6}, u \rightarrow \lambda u$, the part of the metric in the square bracket remains invariant. But the full metric is not indicating that there is hyperscaling violation. In order to find the hyperscaling violation exponent $\theta$, we dimensionally reduce the metric on $S^{2}$ and also on $x^{2}, \ldots, x^{p}$ and express the reduced metric in Einstein frame which is given as,

$$
d s_{9-p, E}^{2}=Q_{1}^{\frac{2}{7-p}} Q_{2} u^{\frac{2(9-p)}{7-p}}\left[-\frac{d t^{2}}{Q_{1} Q_{2}}+\frac{\left(d x^{1}\right)^{2}}{Q_{1} u^{2}}+\frac{\sum_{j=p+1}^{6}\left(d x^{j}\right)^{2}}{Q_{2} u^{2}}+4 \frac{d u^{2}}{u^{2}}\right]
$$

We therefore find that under the above scaling the reduced metric (5) transforms as

$$
\begin{aligned}
d s_{9-p, E} & \rightarrow \lambda^{(9-p) /(7-p)} d s_{9-p, E} \\
& \equiv \lambda^{\theta / d} d s_{9-p, E}
\end{aligned}
$$

where $d=7-p$ is the spatial dimension of the boundary theory and therefore, from (66) we find the hyperscaling violation exponent to have the value $\theta=9-p$. Under the above scaling the other fields transform as,

$$
\phi \rightarrow \phi-\frac{5-p}{2} \log \lambda, \quad A_{[p+1]} \rightarrow \lambda^{2} A_{[2]}, \quad H_{[3]} \rightarrow \lambda H_{[3]}
$$

Therefore, we have shown that the NS5-D $p$ intersecting brane solutions in the near horizon limit do give rise to Lifshitz-like space-time with $z=0, \theta=9-p$ and $d=7-p$. It can be

\footnotetext{
${ }^{6}$ To further support the claim that gravity does decouple for these solutions in the near horizon limit, beyond which we have already discussed in footnote 5, we have studied the scattering of a scalar field minimally coupled to the background (3). The dynamics, in the near horizon limit, is found to be described by a Schrödinger-like equation given as $\left(\partial_{u}^{2}-V(u)\right) \varphi(u)=0$. Here $\varphi$ is related to the scalar field and the scattering potential is given as $V(u)=\left(-4 Q_{1} Q_{2} \omega^{2}+3 / 4+4 \ell(\ell+1)\right) / u^{2}$, where $\omega$ is the energy and $\ell=0,1,2, \ldots$ corresponds to various partial waves. We thus find that for $\omega^{2}<(3 / 16+\ell(\ell+1)) /\left(Q_{1} Q_{2}\right)$, the scalar field experiences an infinite potential barrier at $u \rightarrow 0$ and thus gravity gets decoupled. However, at higher energies when $\omega^{2}$ exceeds the above limit, there is no potential barrier and the gravity may not decouple. The situation is very much like the decoupling in D5 or NS5 brane cases [42].
} 
easily checked that these values of $(z, \theta, d)$ indeed satisfy the following NEC [29],

$$
\begin{aligned}
(d-\theta)(d(z-1)-\theta) & \geq 0 \\
(z-1)(d+z-\theta) & \geq 0
\end{aligned}
$$

Similar Lifshitz-like structures have also been obtained for near horizon geometries of F-D $p$ [1] and also in some intersecting brane solutions [2]. We here give a table for the values of $(z, \theta, d)$ of all these solutions for comparison,

\begin{tabular}{||c|c|c|c||}
\hline \hline Type & $z$ & $\theta$ & $d$ \\
\hline \hline $\begin{array}{c}\text { NS5-D } p \\
1 \leq p \leq 6\end{array}$ & 0 & $9-p$ & $7-p$ \\
\hline $\begin{array}{c}\text { F-D } p \\
0 \leq 5, p \neq 4\end{array}$ & $\frac{2(5-p)}{4-p}$ & $p-\frac{p-2}{4-p}$ & $p+1$ \\
\hline $\begin{array}{l}\text { D0-D4 } \\
\text { D1-D3 } \\
\text { D2-D2 }\end{array}$ & 4 & 2 & 4 \\
\hline $\begin{array}{c}\text { D2-D6 } \\
\text { D3-D5 } \\
\text { D4-D4 }\end{array}$ & 0 & 6 & 4 \\
\hline \hline
\end{tabular}

In the third row, the three intersecting brane solutions D0-D4, D1-D3 and D2-D2' have the same set of values of $(z, \theta, d)$ and similarly, in the 4th row D2-D6, D3-D5 and D4-D4' have the same set of values of $(z, \theta, d)$. Among all the solutions only for F-D2 we have $\theta=d-1$ indicating that the corresponding boundary theory describes compressible metallic states with hidden Fermi surface [24, 25]. The field theoretic meaning of the other scaling solutions are not known.

\section{RG flows and phase structures}

We have seen in the previous section that the dilatons of NS5-D $p$ solutions are in general not constant except for $p=5$. Therefore, as $u$ changes, the dilatons can become large and invalidate the supergravity solutions. Also the curvature of the solutions must remain small for the supergravity description to remain valid. These conditions put some restrictions on the parameter $u$ or the energy parameter in the dual field theory. We will discuss them here in a case by case basis. 


\section{$3.1 \quad$ NS5-D1}

The S-dual of this solution is F-D5 and this case is already discussed in [1] and will not be repeated here. Note that both these solutions have Lifshitz-like structure in the near horizon limit.

\subsection{NS5-D2}

The near horizon geometry in a suitable coordinate $u$ of this solution is given in (4). From there we find that the dilaton remains small only if $u \gg Q_{1}^{1 / 6} Q_{2}^{1 / 3}$ and the curvature remains small if $u \gg 1 /\left(Q_{1}^{1 / 2} Q_{2}\right)$. For large $Q_{1}$ and $Q_{2}$ if the first condition is satisfied then the second one is automatically satisfied. However, when $u \leq Q_{1}^{1 / 6} Q_{2}^{1 / 3}$, the dilaton would be large and in order to get a valid supergravity description we need to uplift the solution to M-theory. The solution in this case takes the form,

$$
d s^{2}=Q_{1}^{\frac{1}{3}} Q_{2}^{\frac{2}{3}}\left[\frac{-d t^{2}+\left(d x^{2}\right)^{2}}{Q_{1} Q_{2} r^{2}}+\frac{\left(d x^{1}\right)^{2}}{Q_{1}}+\frac{\sum_{j=3}^{6}\left(d x^{j}\right)^{2}}{Q_{2}}+\frac{d r^{2}}{r^{2}}+d \Omega_{3}^{2}\right]
$$

The above solution represents the near horizon limit of M5-M2 solutions intersecting on a string along $x^{2}[20$ ]. Note that in writing the solution we have kept the coordinate $r$ (not $u$ ). It is obvious that it has an $\mathrm{AdS}_{3} \times \mathrm{E}^{5} \times \mathrm{S}^{3}$ structure.

\section{$3.3 \quad$ NS5-D3}

The S-dual of this solution is F-D3 and this case is also discussed in [1] and will not be repeated here. Here also both the solutions have Lifshitz-like structure.

\subsection{NS5-D4}

The geometry of this solution in the near horizon limit is given in (4). From the expression of dilaton we find that it will remain small for $u \gg Q_{2} / Q_{1}^{1 / 2}$ and the curvature remains small for $u \gg 1 /\left(Q_{1}^{1 / 2} Q_{2}\right)$. For large $Q_{2}$, the first condition is sufficient for supergravity description to remain valid. However, when $u \leq Q_{2} / Q_{1}^{1 / 2}$, we have to uplift the solution to M-theory. The uplifted solution has the form,

$$
d s^{2}=\left(Q_{1} Q_{2}\right)^{\frac{2}{3}} u^{\frac{4}{3}}\left[\frac{-d t^{2}+\sum_{i=2}^{4}\left(d x^{i}\right)^{2}}{Q_{1} Q_{2}}+\frac{\left(d x^{1}\right)^{2}}{Q_{1} u^{2}}+\frac{\sum_{j=5}^{6}\left(d x^{j}\right)^{2}}{Q_{2} u^{2}}+4 \frac{d u^{2}}{u^{2}}+d \Omega_{2}^{2}+\frac{\left(d x^{11}\right)^{2}}{Q_{1} u^{2}}\right]
$$

The above solution represents the near horizon limit of M5-M5' solution intersecting on a 3-brane along $x^{2}, x^{3}, x^{4}[20]$. This metric again has a Lifshitz-like structure with $(z=0, \theta=$ $6, d=4)$. In obtaining $\theta$ we compactify (10) on $\mathrm{S}^{2}$ and $x^{2,3,4}$ and express the resulting metric in Einstein frame. 


\section{$3.5 \quad$ NS5-D5}

In the near horizon limit NS5-D5 solution expressed in a suitable coordinate $u$ is given in (4). Here the dilaton is constant. However, the dilaton will remain small as long as $Q_{2} \ll Q_{1}$ and the curvature remains small for $u \gg 1 /\left(Q_{1}^{1 / 2} Q_{2}\right)$. But, when $Q_{2} \geq Q_{1}$, the dilaton will become large invalidating the supergravity description. In that case we have to go to the S-dual frame. In the S-dual frame the metric takes the form,

$$
d s^{2}=Q_{1} Q_{2}^{\frac{1}{2}} u\left[\frac{-d t^{2}+\sum_{i=2}^{5}\left(d x^{i}\right)^{2}}{Q_{1} Q_{2}}+\frac{\left(d x^{1}\right)^{2}}{Q_{1} u^{2}}+\frac{\left(d x^{6}\right)^{2}}{Q_{2} u^{2}}+4 \frac{d u^{2}}{u^{2}}+d \Omega_{2}^{2}\right]
$$

Again we find that the above S-dual metric has Lifshitz-like structure with $(z=0, \theta=$ $4, d=2$ ) exactly as those of the original solution.

\subsection{NS5-D6}

The near horizon limit of this solution in a suitable coordinate $u$ is given in (44). Here the dilaton and the curvature of the metric remain small in the region $1 /\left(Q_{1}^{1 / 2} Q_{2}\right) \ll u \ll$ $Q_{1}^{3 / 2} / Q_{2}$. However, when $u \geq Q_{1}^{3 / 2} / Q_{1}$, the dilaton becomes large and the supergravity solution is no longer valid. So, we have to uplift the solution to M-theory. The uplifted solution in this case takes the form,

$$
d s^{2}=Q_{1} Q_{2}^{\frac{2}{3}} u^{\frac{2}{3}}\left[\frac{-d t^{2}+\sum_{i=2}^{6}\left(d x^{i}\right)^{2}}{Q_{1} Q_{2}}+\frac{\left(d x^{1}\right)^{2}}{Q_{1} u^{2}}+4 \frac{d u^{2}}{u^{2}}+d \Omega_{2}^{2}+\frac{\left(d x^{11}-2 Q_{1} \sin ^{2} \frac{\theta}{2} d \phi\right)^{2}}{Q_{1}^{2}}\right]
$$

The above solution represents the near horizon limit of the intersecting M5-KK solution [43]. It can be easily checked that this solution also has Lifshitz-like structure with $(z=0, \theta=$ $3, d=1$ ) as those of the original solution.

\section{Conclusion}

To conclude, in this paper we have constructed 1/4 BPS, threshold intersecting NS5-D $p$ (for $1 \leq p \leq 6$ ) brane solutions of type II string theories starting from the 1/4 BPS threshold F-D5 solution given in [1] of type IIB string theory by applying S- and T-dualities. These solutions are different from the known NS5-D $p$ solutions which are 1/2 BPS and non-threshold. Unlike the known NS5- $\mathrm{D} p$ solutions which in the near horizon limit leads to six dimensional open $\mathrm{D} p$ brane theory on the boundary, the solutions constructed in this paper give Lifshitz-like spacetime, that is, space-time having Lifshitz scaling symmetry along with some hyperscaling violation in the near horizon limit. It is known that some condensed matter system near their quantum critical point show similar kind of scaling symmetry, so, the solutions discussed in 
this paper may describe the gravity dual of such condensed matter systems near that point. Also since the NS5-D $p$ solution we have obtained are 1/4 BPS, the Lifshitz-like solutions should also preserve at least a 1/4 SUSY. We found that the solutions obtained from NS5-D $p$ have a scaling symmetry with dynamical critical exponent $z=0$, the hyperscaling violation exponent $\theta=9-p$ and the spatial dimensions of the boundary theory $d=7-p$. We have seen that these values of $(z, \theta, d)$ satisfy NEC indicating that they might lead to a sensible dual theory. As similar structures were obtained for other type of intersecting solutions we have given a table for the comparison. The dilatons for these solutions are not constant except for $p=5$ and therefore, they give rise to RG flows. We have discussed the various phases of these solutions considering the RG flows. We found that the strongly coupled phases also give Lifshitz-like space-time except for $p=2$. The strongly coupled phase of the near horizon NS5-D2 solution actually has the structure $\mathrm{AdS}_{3} \times \mathrm{E}^{5} \times \mathrm{S}^{3}$. The scaling solutions we have obtained in this paper has dynamical scaling exponent $z=0$ and thus it appears that there is no relaxation in time of the system described by the boundary theory. It would be interesting to understand the field theoretic meaning and the consequences of these scaling solutions.

\section{Acknowledgements}

One of the authors (PD) would like to acknowledge thankfully the financial support of the Council of Scientific and Industrial Research, India (SPM-07/489 (0089)/2010-EMR-I).

\section{References}

[1] P. Dey and S. Roy, "Lifshitz-like space-time from intersecting branes in string/M theory," JHEP 1206129 (2012), [arXiv:1203.5381 [hep-th]].

[2] P. Dey and S. Roy, "Intersecting D-branes and Lifshitz-like space-time," Phys. Rev. D 86, 066009 (2012) arXiv:1204.4858 [hep-th]].

[3] J. M. Maldacena, "The Large N limit of superconformal field theories and supergravity," Adv. Theor. Math. Phys. 2, 231 (1998) [Int. J. Theor. Phys. 38, 1113 (1999)] hep-th/9711200.

[4] S. S. Gubser, I. R. Klebanov and A. M. Polyakov, "Gauge theory correlators from noncritical string theory," Phys. Lett. B 428, 105 (1998) hep-th/9802109.

[5] E. Witten, "Anti-de Sitter space and holography," Adv. Theor. Math. Phys. 2, 253 (1998) hep-th/9802150. 
[6] O. Aharony, S. S. Gubser, J. M. Maldacena, H. Ooguri and Y. Oz, "Large N field theories, string theory and gravity," Phys. Rept. 323, 183 (2000) hep-th/9905111.

[7] N. Itzhaki, J. M. Maldacena, J. Sonnenschein and S. Yankielowicz, "Supergravity and the large N limit of theories with sixteen supercharges," Phys. Rev. D 58, 046004 (1998) hep-th/9802042.

[8] J. G. Russo and A. A. Tseytlin, "Waves, boosted branes and BPS states in m theory," Nucl. Phys. B 490, 121 (1997) hep-th/9611047.

[9] J. C. Breckenridge, G. Michaud and R. C. Myers, "More D-brane bound states," Phys. Rev. D 55, 6438 (1997) hep-th/9611174.

[10] M. S. Costa and G. Papadopoulos, "Superstring dualities and p-brane bound states," Nucl. Phys. B 510, 217 (1998) hep-th/9612204.

[11] J. X. Lu and S. Roy, "Nonthreshold (f, Dp) bound states," Nucl. Phys. B 560, 181 (1999) hep-th/9904129.

[12] J. X. Lu and S. Roy, "An SL(2,Z) multiplet of type IIB super five-branes," Phys. Lett. B 428, 289 (1998) hep-th/9802080.

[13] M. Alishahiha, Y. Oz and J. G. Russo, "Supergravity and light - like noncommutativity," JHEP 0009, 002 (2000) hep-th/0007215]; M. Alishahiha and Y. Oz, "Supergravity and 'new' six-dimensional gauge theories," Phys. Lett. B 495, 418 (2000) hep-th/0008172.

[14] I. Mitra and S. Roy, "(NS5,Dp) and (NS5,D (p+2),Dp) bound states of type IIB and type IIA string theories," JHEP 0102, 026 (2001) hep-th/0011236.

[15] J. M. Maldacena and J. G. Russo, "Large N limit of noncommutative gauge theories," JHEP 9909, 025 (1999) hep-th/9908134.

[16] A. Hashimoto and N. Itzhaki, "Noncommutative Yang-Mills and the AdS / CFT correspondence," Phys. Lett. B 465, 142 (1999) hep-th/9907166.

[17] N. Seiberg, L. Susskind and N. Toumbas, "Strings in background electric field, space / time noncommutativity and a new noncritical string theory," JHEP 0006, 021 (2000) hep-th/0005040.

[18] R. Gopakumar, J. M. Maldacena, S. Minwalla and A. Strominger, "S duality and noncommutative gauge theory," JHEP 0006, 036 (2000) hep-th/0005048.

[19] R. Gopakumar, S. Minwalla, N. Seiberg and A. Strominger, "(OM) theory in diverse dimensions," JHEP 0008, 008 (2000) hep-th/0006062. 
[20] A. A. Tseytlin, "Composite BPS configurations of p-branes in ten-dimensions and eleven-dimensions," Class. Quant. Grav. 14, 2085 (1997) hep-th/9702163.

[21] J. P. Gauntlett, D. A. Kastor and J. H. Traschen, "Overlapping branes in M theory," Nucl. Phys. B 478, 544 (1996) hep-th/9604179]; J. P. Gauntlett, "Intersecting branes," In *Seoul/Sokcho 1997, Dualities in gauge and string theories* 146-193 hep-th/9705011.

[22] K. Behrndt, E. Bergshoeff and B. Janssen, "Intersecting d-branes in ten-dimensions and six-dimensions," Phys. Rev. D 55, 3785 (1997) hep-th/9604168].

[23] D. S. Fisher, "Scaling and critical slowing down in random-field Ising systems," Phys. Rev. Lett. 56, 416 (1986).

[24] N. Ogawa, T. Takayanagi and T. Ugajin, "Holographic Fermi Surfaces and Entanglement Entropy," JHEP 1201, 125 (2012) [arXiv:1111.1023 [hep-th]].

[25] L. Huijse, S. Sachdev and B. Swingle, "Hidden Fermi surfaces in compressible states of gauge-gravity duality," Phys. Rev. B 85, 035121 (2012) arXiv:1112.0573 [cond-mat.strel]].

[26] C. Charmousis, B. Gouteraux, B. S. Kim, E. Kiritsis and R. Meyer, "Effective Holographic Theories for low-temperature condensed matter systems," JHEP 1011, 151 (2010) arXiv:1005.4690 [hep-th]].

[27] B. Gouteraux and E. Kiritsis, "Generalized Holographic Quantum Criticality at Finite Density," JHEP 1112, 036 (2011) [arXiv:1107.2116 [hep-th]].

[28] E. Shaghoulian, "Holographic Entanglement Entropy and Fermi Surfaces," JHEP 1205, 065 (2012) arXiv:1112.2702 [hep-th]].

[29] X. Dong, S. Harrison, S. Kachru, G. Torroba and H. Wang, "Aspects of holography for theories with hyperscaling violation," JHEP 1206, 041 (2012) arXiv:1201.1905 [hepth]].

[30] K. Narayan, "On Lifshitz scaling and hyperscaling violation in string theory," Phys. Rev. D 85, 106006 (2012) arXiv:1202.5935 [hep-th]].

[31] B. S. Kim, "Schródinger Holography with and without Hyperscaling Violation," JHEP 1206, 116 (2012) arXiv:1202.6062 [hep-th]].

[32] H. Singh, "Lifshitz/Schródinger Dp-branes and dynamical exponents," JHEP 1207, 082 (2012) arXiv:1202.6533 [hep-th]]. 
[33] E. Perlmutter, "Hyperscaling violation from supergravity," JHEP 1206, 165 (2012) arXiv:1205.0242 [hep-th]].

[34] M. Cadoni and S. Mignemi, "Phase transition and hyperscaling violation for scalar Black Branes," JHEP 1206, 056 (2012) [arXiv:1205.0412 [hep-th]].

[35] M. Ammon, M. Kaminski and A. Karch, "Hyperscaling-Violation on Probe D-Branes," arXiv:1207.1726 [hep-th].

[36] J. Bhattacharya, S. Cremonini and A. Sinkovics, "On the IR completion of geometries with hyperscaling violation," arXiv:1208.1752 [hep-th].

[37] P. Dey and S. Roy, "Holographic entanglement entropy of the near horizon 1/4 BPS F-D $p$ bound states," arXiv:1208.1820 [hep-th].

[38] N. Kundu, P. Narayan, N. Sircar and S. P. Trivedi, "Entangled Dilaton Dyons," arXiv:1208.2008 [hep-th].

[39] M. Alishahiha and H. Yavartanoo, "On Holography with Hyperscaling Violation," arXiv:1208.6197 [hep-th].

[40] S. Sachdev, "Quantum Phase Transitions," Cambridge University Press, 1999.

[41] S. Sachdev, "The quantum phases of matter," arXiv:1203.4565 [hep-th].

[42] M. Alishahiha, H. Ita and Y. Oz, "Graviton scattering on D-6 branes with B fields," JHEP 0006, 002 (2000) hep-th/0004011.

[43] E. Bergshoeff, M. de Roo, E. Eyras, B. Janssen and J. P. van der Schaar, "Intersections involving monopoles and waves in eleven-dimensions," Class. Quant. Grav. 14, 2757 (1997) [hep-th/9704120]. 